Check for updates

Cite this: RSC Adv., 2018, 8, 1427

Received 29th August 2017

Accepted 18th December 2017

DOI: $10.1039 / \mathrm{c} 7 \mathrm{ra09564c}$

rsc.li/rsc-advances

\section{Photolysis of sulfamethazine using UV irradiation in an aqueous medium}

\author{
Zhigang Yi, (D)*a Juan Wang, ${ }^{\text {b }}$ Qiong Tang ${ }^{\mathrm{a}}$ and Tao Jiang ${ }^{\mathrm{a}}$
}

\begin{abstract}
Although many studies have been focused on the photochemistry of antibiotics, the roles of reactive species in photolysis and the effects of dissolved substances on antibiotic photochemical behavior have been poorly examined. The photolytic behaviors of sulfamethazine (SMN) in pure water were investigated via adding different scavengers to quench the active species. Results showed that decomposition of the tripletexcited state of SMN ( $\left.{ }^{3} \mathrm{SMN} *\right)$ by direct photolysis was the main path of SMN photolysis in water. Moreover, self-sensitized SMN cannot be ignored during SMN photodegradation. The main photoproducts of SMN were identified by LC-MS/MS, which indicated that SMN could not be mineralized although the photolysis under UV was effective. The effects of $\mathrm{Cl}^{-}, \mathrm{NO}_{3}{ }^{-}$, and fulvic acid (FA) (common substances in natural water) on SMN photolytic behaviors were also studied. The triplet-induced halogenation of SMN increases the ionic strength and reduces the ground state SMN; these are the primary causes of promotion of SMN photolysis by $\mathrm{Cl}^{-}$. More ${ }^{\circ} \mathrm{OH}$ produced in the presence of $\mathrm{NO}_{3}{ }^{-}$could promote $\mathrm{SMN}$ photolysis. Competitive absorption of photons of FA with SMN and ROS scavenged by FA were the main reasons for the inhibition of SMN photolysis. The research findings are helpful for further studies on the environmental risks of ACs in natural waters and promoting the development of AC pollution treatment technology.
\end{abstract}

\section{Introduction}

In the last few decades, large amounts of veterinary antibiotics (ACs) have been used in animal husbandry as therapeutic medicine and feed additives for growth promotion. Moreover, one of the most popular groups of ACs, which are mainly used in livestock, are sulfonamides (SNs). ${ }^{\mathbf{1}, 2}$ In China, AC use in stock raising was nearly 92700 tons, and usage of SNs was nearly 7920 tons in $2013 .^{3}$ In the USA, 16000 tons of ACs, including $2.3 \%$ SNs, were consumed per year. In Europe, the output of SNs ranged from $11 \%$ to $23 \%$ of the total production of ACs. ${ }^{4} \mathrm{SNs}$ may be excreted from the body in their parent form without absorption and metabolism by animals. Because of their high stability and solubility, conventional sewage disposal technology used in livestock farming is not efficient for SN removal. The average removal ratio of SNs by activated sludge was found to be $24 \%,{ }^{1}$ which meant that a mass of these drugs was introduced into the biosphere every year. ${ }^{\mathbf{1 , 5}}$ In this case, ACs were detected in rivers, lakes, estuarine, and coastal waters many years ago. ${ }^{6-8}$ The highest concentration of sulfamethazine (SMN) in manure of swine farms in South China was up to $0.250 \mathrm{mg} \mathrm{kg}{ }^{-1}$. ${ }^{9}$ The concentration of ACs in the aquatic envi-

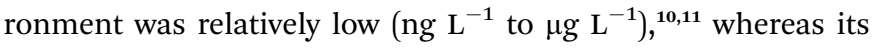

${ }^{a}$ College of Chemistry, Leshan Normal University, Leshan, Sichuan 614004, China. E-mail: yizhigang117@hotmail.com

${ }^{b}$ Environmental Monitoring Station of Environmental Protection Bureau of Rizhao Lanshan, Lanshan, Shandong 276800, China presence in AC manufacturing effluents might reach $\mathrm{mg} \mathrm{L}^{-1}$ levels. ${ }^{12}$ The bacterial resistance and photo-modified toxicities of ACs could cause severe negative impact on human health and the environment. ${ }^{\mathbf{1 1 3 - 1 8}}$ ACs have been classified as particularly dangerous pollutants for the environment. ${ }^{1}$

The obstinate nature of ACs residues interferes with the removal of these compounds from the environment by traditional biological treatments. Finding effective methods to eliminate the ACs residues is required for environmentally sustainable development. Heterogeneous photocatalysis, one of the typical advanced oxidation techniques (AOPs), is an efficient treatment to promote degradation of organic pollutants. ${ }^{19}$ Further, the performance of photocatalytic technology could be effectively improved via photocatalyst modification, ${ }^{20}$ electrochemical, microwave or ultrasonic-assisted photocatalytic technology. ${ }^{21-23}$ The efficiency of photocatalysis is based on the formation of reactive species through the irradiation of light energy on catalysts. During photocatalytic degradation of organic contaminants, photodegradation may play an important role, and photochemical decomposition has also been proven to be a major transformation pathway for organic pollutants in surface waters. ${ }^{24,25}$ Therefore, it is significant to carry out a study on photodecomposition of pollutants.

Photolysis of ACs is mainly caused by ultraviolet wavelength irradiation, and ACs can undergo not only direct or indirect photolysis, but also self-sensitized photo-oxidation. ${ }^{2}$ During direct photolysis, photon absorption promotes electrons from the initial ground state of ACs to produce electronically excited 
species in the singlet state (AS*) or triplet state $\left({ }^{3} \mathrm{AS}^{*}\right)$. On the one hand, the excited species can be decomposed into photoproducts. On the other hand, by self-sensitization, ${ }^{3} \mathrm{AS}^{*}$ can transfer the energy to the ground state of ${ }^{3} \mathrm{O}_{2}$ or $\mathrm{H}_{2} \mathrm{O}$ to form reactive oxygen species (ROS, e.g., ${ }^{\circ} \mathrm{OH}$ and ${ }^{1} \mathrm{O}_{2}$ ) ${ }^{26,27}$ In addition to the direct and self-sensitized photolysis, indirect photolysis is a significant elimination pathway for many aquatic pollutants; it is initiated by ROS, formed via optical absorption by photosensitizers (e.g., dissolved organic matter (DOM), Fe species, halide ions, and $\left.\mathrm{H}_{2} \mathrm{O}_{2}\right) \cdot{ }^{28-30}$ However, controversy still exists about which path should play the leading role in different research. Studies indicated that many byproducts were still retained during the photolysis of antibiotics, the intermediates showed an increasing toxicity, and the solutions after photolysis still had certain residual antibacterial activity. ${ }^{31-35}$ Because the photolytic byproducts will be different due to different photolytic reaction paths of ACs, studies on the main factors for photolysis and the reaction paths of ACs under UV irradiation are helpful for a further study on the environmental risks of ACs in natural waters and promoting the development of AC pollution treatment technology.

In this study, we employed SMN as a proxy AC to investigate photolytic behavior under UV irradiation and detect the role of triplet-excited state and ROS in the photodegradation of ACs. Furthermore, the photodecomposition pathway of SMN was proposed. The influence of common dissolved substances in natural water on the photodecomposition of SMN was also discussed in this study.

\section{Materials and methods}

\subsection{Chemicals}

High purity standard SMN (99\%), used as a target pollutant in photochemical experiments and as a standard in highperformance liquid chromatography (HPLC) analysis, was purchased from Aladdin Industrial Corporation (Shanghai, China). Acetonitrile and isopropanol were of HPLC grade and obtained from Chengdu Best Reagent (Chengdu, China). Sorbic acid ( $t, t$-HDA, 99\%), sodium chloride, sodium nitrate, fulvic acid (FA), and other chemical reagents were of analytical grade and obtained from Chengdu Best Reagent. Ultrapure water was used to prepare SMN solutions and HPLC eluent.

\subsection{Experimental set-up}

A merry-go-round photochemical reactor with a magnetic stirrer (illustrated in Fig. 1) was employed. The high pressure Hg lamp was in a quartz sleeve and located at the center of the reactor. The temperature of the reaction system was $25^{\circ} \mathrm{C}$. The $50 \mathrm{~mL}$ SMN solutions were put in quartz tubes around and equidistant from the Hg lamp, irradiated under UV over a period of $60 \mathrm{~min}$, and aliquots were withdrawn for analysis at scheduled time intervals. To investigate the hydrolytic degree of SMN during photolysis, non-irradiated experiments were conducted simultaneously. The SMN solutions were wrapped in an aluminum foil, and other conditions were kept the same as those for the unwrapped samples. All experiments were performed in triplicate.

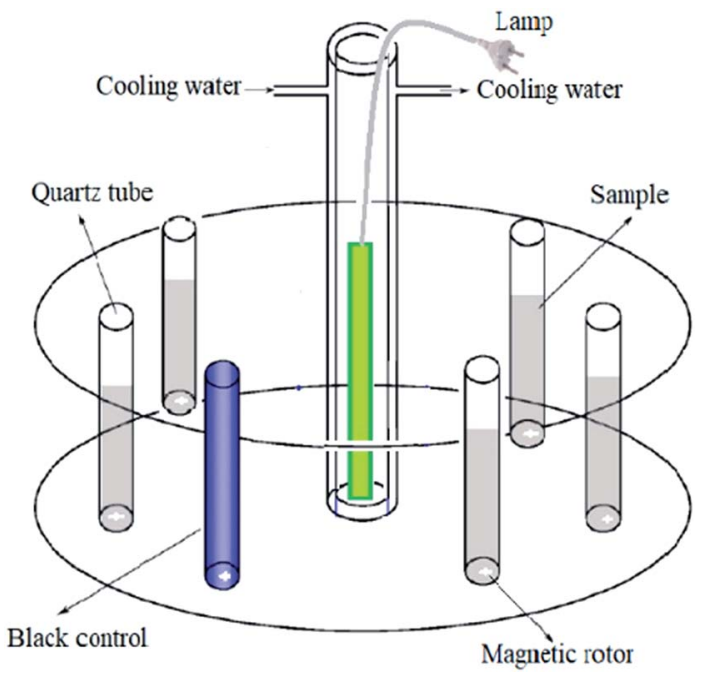

Fig. 1 A schematic of the experimental setup.

\subsection{Analytical methods}

The concentration of SMN at different irradiation times was determined by HPLC (LC-2010HT, Shimadzu) coupled with UV/ VIS detection (LC-UV/VIS). The parameters of the analysis were set according to the research of Batista et al. ${ }^{2} \mathrm{a} \mathrm{C}_{18} 5 \mu \mathrm{m}$ to $100 \AA$ column $(250 \mathrm{~mm} \times 4.60 \mathrm{~mm})$. The detection wavelength of SMN was found to be at $268 \mathrm{~nm}$. The sample injection volume was $50.0 \mu \mathrm{L}$. The eluents were (A) $\mathrm{H}_{2} \mathrm{O}+0.200 \%$ acetic acid and (B) acetonitrile at $80: 20$ ratio, and the flow rate was set at $1.00 \mathrm{~mL} \mathrm{~min}^{-1}$. The detection and quantification limits were $0.170 \mathrm{mg} \mathrm{L}^{-1}$ and $0.500 \mathrm{mg} \mathrm{L}^{-1}$, respectively.

The products of photolysis were identified by a Shimadzu LC-20A liquid chromatography system coupled with a Shimadzu LCMS-8030 triple quadrupole mass spectrometer (LCMS/MS). The eluent and column used were the same as those of LC-UV/VIS, but the ratio of A and B eluents was altered during the analysis: it started with $10 \%$ of B, then it increased to $60 \%$ after $10 \mathrm{~min}$, then to $90 \%$ in another $8 \mathrm{~min}$, and finally the content of B dropped to $10 \%$ in another $10 \mathrm{~min}$ and remained at this ratio until the end of the run. The detection was performed using an electrospray ionization (ESI) source operating in the positive mode. The parameters were set as follows: capillary voltage $4000 \mathrm{~V}$, drying gas temperature $300{ }^{\circ} \mathrm{C}$, drying gas flow

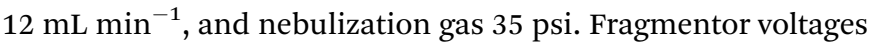
were adjusted between 10 and $30 \mathrm{~V}$ to obtain precursor ions of degradation products.

The total organic carbon (TOC) was detected by a TOC analyzer (TOC4100, Shimadzu).

\section{Results and discussions}

\subsection{The determination of initial concentration of SMN and the light source}

The concentration of SMN in the aquatic environment (ng L $\mathrm{L}^{-1}$ to $\mu \mathrm{g} \mathrm{L}^{-1}$ ) is relatively low. A pre-concentration step is required prior to quantification using HPLC. ${ }^{36,37}$ Although 
previous research has reported that the solid phase extraction (SPE) technique can improve the sensitivity of the analytical methods by HPLC, the use of SPE for quantification of sulfonamides in aqueous samples has not been examined in detail. ${ }^{36}$ During photodegradation of ACs, different initial concentrations of ACs caused different amounts of reactant molecules to be photo-excited; this affected the rate of photolysis, but had no impact on the reaction mechanism. ${ }^{2,6}$ Furthermore, Nassar et $a l .{ }^{38}$ evaluated the photodecomposition of four different ACs under irradiation of sunlight and a single wavelength UV source $(254 \mathrm{~nm})$. The results showed that UV irradiation was effective to degrade these drugs, and photolysis of ACs under simulated solar irradiation was caused by AC absorbing the UV light from sunlight.

Due to all the abovementioned reasons, the selected initial concentration of SMN and the light source in this study were $20 \mathrm{mg} \mathrm{L}^{-1}$ and a $300 \mathrm{~W}$ high pressure $\mathrm{Hg}$ lamp, respectively. Although these two parameters were significantly different from the antibiotic environment in natural water bodies, the conclusions of this study could provide theoretical reference for the photochemical behavior of antibiotic residues in natural aquatic environments.

\subsection{Photolysis of SMN in pure water}

To investigate the photolytic process of SMN, we explored SMN photolysis in pure water under $300 \mathrm{~W}$ UV radiation. The experiments have been carried out at $\mathrm{pH} 7$ at which the neutral form of SMN dominates $(>80 \%) .{ }^{39}$ Previous studies found that photo-excited pharmaceutical compounds, formed by the photosensitization process, interacted with molecular oxygen to form ${ }^{\circ} \mathrm{OH},{ }^{1} \mathrm{O}_{2}$, and $\mathrm{O}_{2}{ }^{\cdot-} \cdot{ }^{40}$ During the photolytic experiment, we selected 2-propanol, sodium azide $\left(\mathrm{NaN}_{3}\right)$, and $p$-quinone as scavengers to quench ${ }^{\circ} \mathrm{OH},{ }^{1} \mathrm{O}_{2}$, and $\mathrm{O}_{2}{ }^{--}$respectively. ${ }^{41} \mathrm{SMN}$ degradation and the logarithm of relative SMN concentration versus irradiation time are displayed in Fig. 2. SMN photolysis followed pseudo-first-order kinetics in pure water. The degradation ratio of SMN achieved a value of $78 \%$ after 60 min irradiation, and the degradation rate constant $(k)$ of SMN was $2.58 \times 10^{-2} \mathrm{~min}^{-1}$ in pure water. The values of $k$ decreased to $2.19 \times 10^{-2} \mathrm{~min}^{-1}, 2.33 \times 10^{-2} \mathrm{~min}^{-1}$, and $2.37 \times 10^{-2} \mathrm{~min}^{-1}$ and the degradation ratios were $72 \%, 74 \%$, and $75 \%$ in the presence of 2-propanol, $\mathrm{NaN}_{3}$, and $p$-quinone, respectively. The results indicated that ${ }^{\circ} \mathrm{OH},{ }^{1} \mathrm{O}_{2}$, and $\mathrm{O}_{2}{ }^{-}$- were formed during SMN photolysis in pure water via the photosensitization process of SMN, and the impact of ${ }^{\circ} \mathrm{OH}$ was more noticeable than that of ${ }^{1} \mathrm{O}_{2}$ and $\mathrm{O}_{2}{ }^{-}$on the photolysis of SMN, which were consistent with the early research conclusions that hydroxyl radicals played a critical role in the photochemical transformation of organic pollutants. ${ }^{\mathbf{4 2 - 4 4}}$ However, the ratio and rate constants $(k)$ of SMN molecules had no evident decline after adding scavengers for ${ }^{\circ} \mathrm{OH},{ }^{1} \mathrm{O}_{2}$, and $\mathrm{O}_{2}{ }^{\cdot-}$; this implied that direct photolysis was the main path for photochemical behavior of SMN in pure water.

To further understand the effect of self-sensitized behavior on SMN photolytic reactions, comparative trials under deoxygenation by $\mathrm{N}_{2}$-sparging, oxygen filling, and adding sorbic acid as a scavenger to quench ${ }^{3} \mathrm{SMN}^{*}$ were carried out in SMN photolysis. The results are also shown in Fig. 2. The value of $k$ increased to $3.31 \times 10^{-2} \mathrm{~min}^{-1}$ from $2.75 \times 10^{-2} \mathrm{~min}^{-1}$ after deoxygenation, but decreased to $1.99 \times 10^{-2} \mathrm{~min}^{-1}$ and $1.60 \times$ $10^{-2} \min ^{-1}$ after oxygen filling and addition of sorbic acid, respectively (Fig. 2). Removal of ${ }^{3} \mathrm{O}_{2}$ from solution by $\mathrm{N}_{2}$ sparging could lead to a decreased formation rate of ${ }^{1} \mathrm{O}_{2}$ as well as lower quenching rate of ${ }^{3} \mathrm{SMN}^{*}$, and oxygen filling was an opposite process to strengthen the self-sensitization. Based on these results, we could state that the self-sensitized behavior had an inhibiting effect on SMN photolysis. The reduction of $k$ after adding sorbic acid further demonstrated that the steady state concentration of ${ }^{3} \mathrm{SMN}^{*}$ was very important during SMN photolysis, and the direct decomposition of ${ }^{3} \mathrm{SMN}^{*}$ was the main pathway of SMN photolysis.

To investigate the hydrolysis of SMN, non-irradiated experiments were conducted simultaneously. No obvious loss of SMN was observed in pure water solution of SMN; this implied that hydrolysis of SMN was negligible during the SMN aquatic environmental behavior.

\subsection{Pathways of SMN photolysis in pure water}

In our experiment, we found that the main path of formation of SMN photoproducts in water was ${ }^{3} \mathrm{SMN}^{*}$ decomposition by
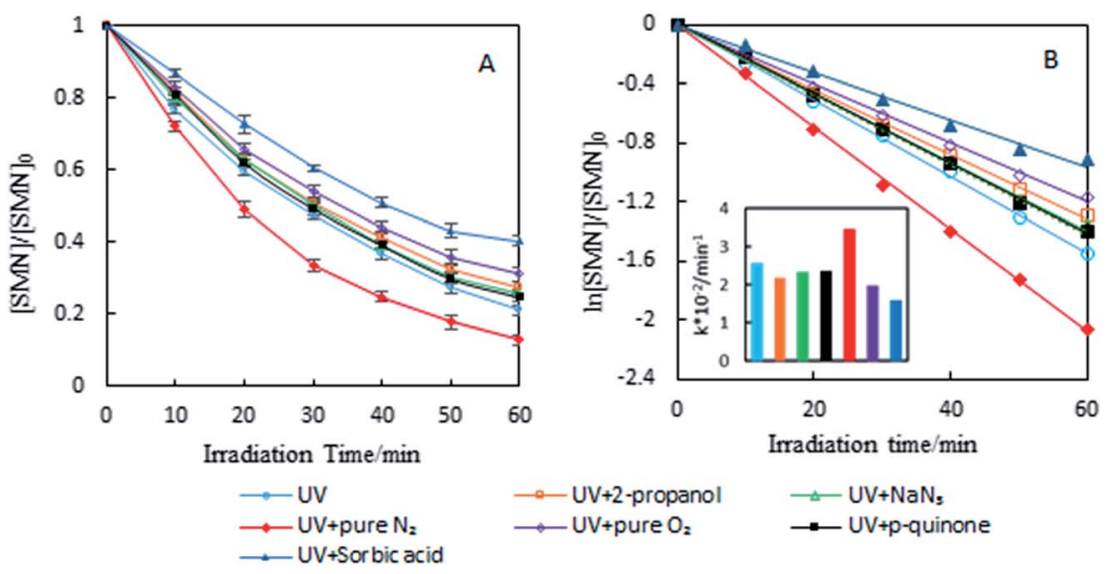

Fig. 2 Degradation ratio (A) and kinetics (B) of SMN photolysis under different conditions. 


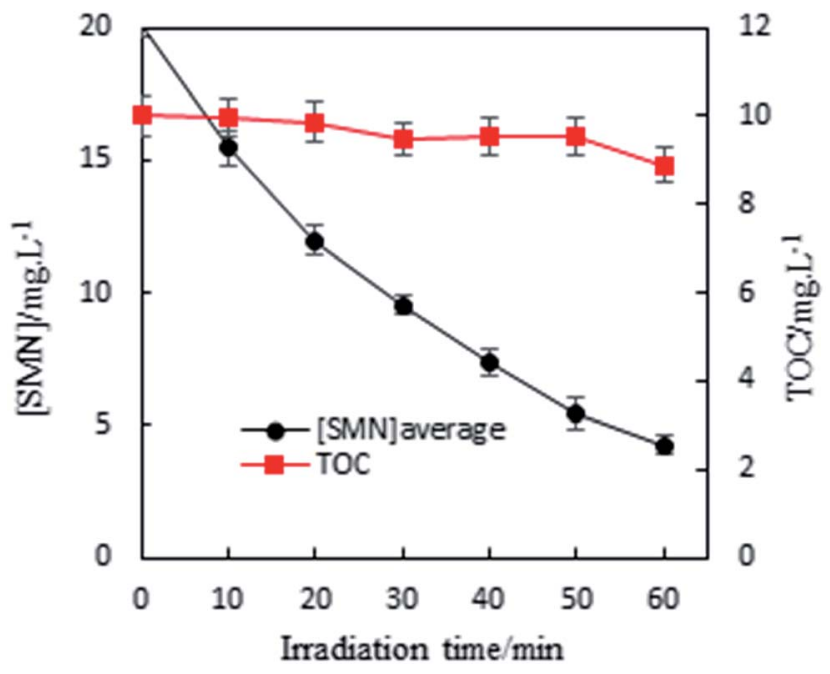

Fig. 3 TOC with the SMN photolysis under UV irradiation.

direct photolysis. Moreover, species of ROS formed by selfsensitized SMN could degrade SMN, which could not be ignored during SMN photochemical behaviors. To determine the mineralization degree of SMN and identify the products of SMN photolysis, TOC was monitored along with SMN photolysis under UV irradiation. The solutions obtained after $60 \mathrm{~min}$ of SMN photodecomposition were analyzed using LC-MS/MS. As shown in Fig. 3, no significant removal of TOC was observed even after $60 \mathrm{~min}$; this meant that most of the organic carbon was still retained although the SMN photolysis under UV was effective.

The specific ion mass spectra of major intermediates are depicted in Fig. 4. There were three main photoproducts that could be observed: SMN-1, SMN-2, and SMN-3 with $m / z$ of 124 , 215 , and 295 , respectively. ${ }^{\circ} \mathrm{OH}$ radicals formed during the selfsensitization process of SMN attacked the benzene ring or the dimethyl pyrimidine group of $\mathrm{SMN}$; this resulted in the formation of SMN-3. SMN-2 was derived from $\mathrm{SO}_{2}$ removal from SMN. SMN-1 was produced from the broken carbon-nitrogen bond via intermediate photolysis. These results were consistent with the previous research on photolytic mechanism of sulfonamide antibiotics. ${ }^{2,38}$ The proposed pathways of SMN photolysis in pure water are displayed in Scheme 1.

\subsection{Effect of soluble substances on SMN photolysis}

Halide ions, nitrate, and dissolved organic matter (DOM) are ordinary soluble substances in natural waters, which can be converted into free radicals under sunlight. ${ }^{6,45}$ Indirect photolytic behaviors of SMN caused by soluble substances are common photodecomposition behavior of SMN. We selected $\mathrm{Cl}^{-}, \mathrm{NO}_{3}{ }^{-}$, and $\mathrm{FA}$ as probes to explore the effect of photoactive substances on SMN photolysis in water. In view of SMN having been frequently detected in both freshwater and seawater, the concentrations of $\mathrm{Cl}^{-}, \mathrm{NO}_{3}{ }^{-}$, and $\mathrm{FA}$ were set according to their content in natural waters (Table 1$).^{6}$

As the content of $\mathrm{Cl}^{-}$is very low in freshwater and exhibits gradient variation in estuarine waters, we set different $\mathrm{Cl}^{-}$ concentrations $(100,300$, and $500 \mathrm{mM})$ relevant to estuarine and sea conditions ${ }^{6}$ to explore the effects on the photolysis of SMN. Experiments showed that the $k$ values of SMN photolysis increased with an increase in $\mathrm{Cl}^{-}$concentration (Fig. 5). In pure water, the main photolysis pathway is ${ }^{3} \mathrm{SMN}^{*}$ disintegration to photoproducts by direct photolysis. Hence, the steady state concentration of triplet-excited state $\left(\mathrm{T}_{\mathrm{ss}}\right)$ is very important for degradation. Previous studies indicated the $\mathrm{T}_{\mathrm{ss}}$ of ${ }^{3} \mathrm{SAs} *$ was higher at a higher ionic strength. ${ }^{46,47} E_{\mathrm{T}}$ was employed to evaluate the electron accepting ability of the ${ }^{3} \mathrm{SAs}^{*} \cdot{ }^{48,49} \mathrm{SMN}$ exists in natural water $(\mathrm{pH}=6.5-8.5)$ as two different species: neutral state $\left(\mathrm{SMN}^{0}\right)$ and anionic state $\left(\mathrm{SMN}^{-}\right){ }^{39}$ The $E_{\mathrm{T}}$ values are $2.45 \mathrm{~V}$ and $2.25 \mathrm{~V}$, which let ${ }^{3} \mathrm{SMN}^{*}$ oxidize $\mathrm{Cl}^{-}\left[E\left(\mathrm{Cl}_{2}{ }^{-} / \mathrm{Cl}^{-}\right)=\right.$ $2.0 \mathrm{~V}] .{ }^{6}$ Thus, the deactivation of ${ }^{3} \mathrm{SMN}^{*}$ induced by $\mathrm{Cl}^{-}$may lead to the formation of $\mathrm{Cl}_{2}{ }^{--}$and halogenated intermediates via the pathway demonstrated in Scheme 2. Triplet-induced halogenation of SMN will increase the ionic strength effect, and at the same time reduce the ground state SMN; this can explain why the rate of SMN photolysis is faster in the presence of $\mathrm{Cl}^{-}$as compared to that in the presence of pure water.

The role of $\mathrm{NO}_{3}{ }^{-}$was paradoxical: either its absorption (200-400 $\mathrm{nm})$ covering the SMN absorption range $(200-330 \mathrm{~nm})$ resulted in a decrease of photolytic rate of SMN or produced reactive species (e.g., ${ }^{\circ} \mathrm{OH}, \mathrm{NO}_{2}{ }^{\circ}$ ) via sensitization effects (eqn (1)-(3)); this could increase the photolytic rate of SMN.

$$
\begin{gathered}
\mathrm{NO}_{3}{ }^{-} \stackrel{h v}{\longrightarrow}\left[\mathrm{NO}_{3}{ }^{-}\right]^{*} \\
{\left[\mathrm{NO}_{3}{ }^{-}\right]^{*} \rightarrow \mathrm{NO}_{2}{ }^{-}+\mathrm{O}^{-} \stackrel{\mathrm{H}_{2} \mathrm{O}}{\longrightarrow} \mathrm{NO}_{2}+{ }^{\circ} \mathrm{OH}+\mathrm{OH}^{-}} \\
{\left[\mathrm{NO}_{3}{ }^{-}\right]^{*} \rightarrow \mathrm{NO}_{2}{ }^{-}+\mathrm{O}\left(^{3} \mathrm{P}\right)}
\end{gathered}
$$
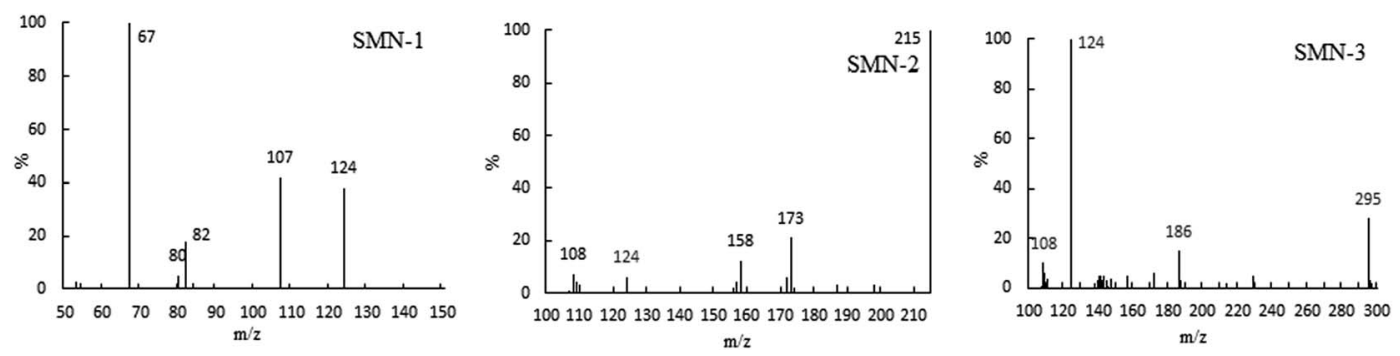

Fig. 4 Major photolytic intermediate ion mass spectra of SMN. 


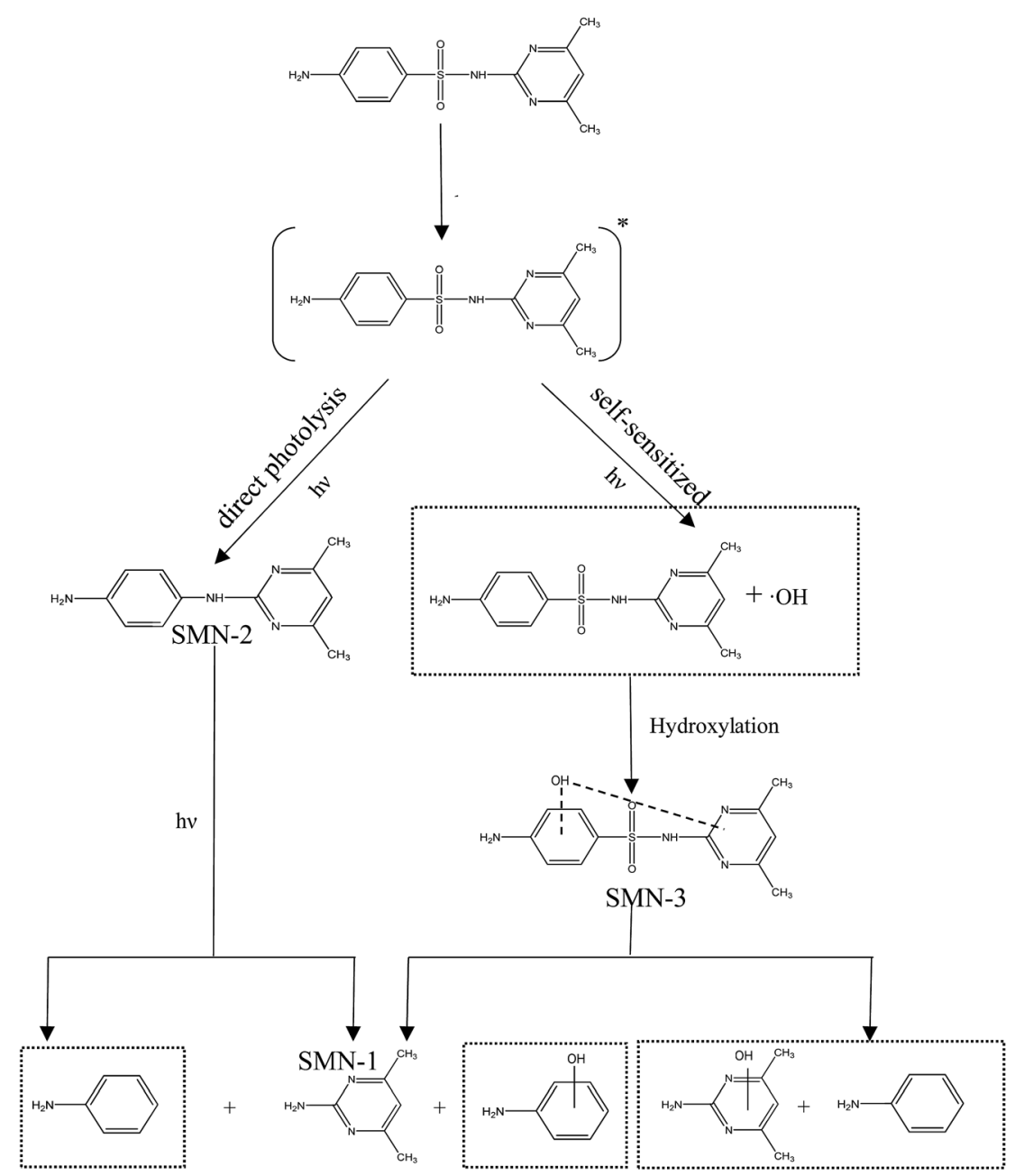

Scheme 1 Proposed degradation pathways of SMN in pure water.

Table 1 Concentrations of $\mathrm{Cl}^{-}, \mathrm{NO}_{3}^{-}$, and $\mathrm{TOC}$ for freshwater and seawater

\begin{tabular}{lcc}
\hline Parameters & Freshwater & Seawater \\
\hline $\mathrm{Cl}^{-}(\mathrm{mM})$ & 3.4 & 469 \\
$\mathrm{NO}_{3}{ }^{-}(\mu \mathrm{M})$ & 93.6 & 29.0 \\
$\mathrm{TOC}^{\left(\mathrm{mg} \mathrm{C} \mathrm{L}{ }^{-1}\right)}$ & 4.0 & 6.1
\end{tabular}

Our experimental results are shown in Fig. 6. In the concentration range of $\mathrm{NO}_{3}{ }^{-}$in natural water, the existence of $\mathrm{NO}_{3}{ }^{-}$led to the increase of SMN photolytic rate. To know whether ${ }^{\circ} \mathrm{OH}$ formed by $\mathrm{NO}_{3}{ }^{-}$sensitization played an important role during SMN photolysis, we added 2-propanol to the reaction system; the experimental findings are exhibited in Fig. 7. The $k$ value of SMN photolysis decreased than that in pure water. The experiment confirmed that more production of ${ }^{\circ} \mathrm{OH}$ in the presence of $\mathrm{NO}_{3}{ }^{-}$as compared to that in pure water was the main cause of $\mathrm{NO}_{3}{ }^{-}$promoting the SMN photolysis.

FA is a ubiquitous DOM in natural waters that has strong photochemical activity. In this study, following the concentration of DOM in natural waters, ${ }^{50}$ different concentrations of FA
(3.0, 6.0, and 9.0 $\mathrm{mg} \mathrm{C} \mathrm{L}^{-1}$ ) were added to pure water to investigate the effect of DOM on the photolysis of SMN. As shown in Fig. 8, photolysis rate constants of SMN decreased with an increase in FA concentration.

These conclusions were not consistent with previous studies. Others found that FA formed the excited state ${ }^{3} \mathrm{FA}^{*}$ and ROS under irradiation, which further induced the degradation of organic compounds and was a significant promoter of organic compound photolysis. ${ }^{51}$ The photolysis pathway of FA in SMN solution is displayed in eqn (4)-(6).

$$
\begin{gathered}
\mathrm{FA} \stackrel{{ }^{h v}}{\longrightarrow}{ }^{3} \mathrm{FA}^{*} \\
{ }^{3} \mathrm{FA}^{*}+\mathrm{O}_{2} \stackrel{\mathrm{H}_{2} \mathrm{O}}{\longrightarrow} \mathrm{FA}+\mathrm{ROS} \\
\mathrm{FA}+\mathrm{ROS} \rightarrow \text { photolytic products }
\end{gathered}
$$

In our case, the inhibiting effect of FA on SMN photolysis might have two factors: one, the steady state concentration of ${ }^{3} \mathrm{SMN}^{*}$ was very important for SMN photolysis; the direct decomposition of ${ }^{3} \mathrm{SMN}^{*}$ has been proven to be the main pathway of SMN photolysis. Therefore, the competitive 

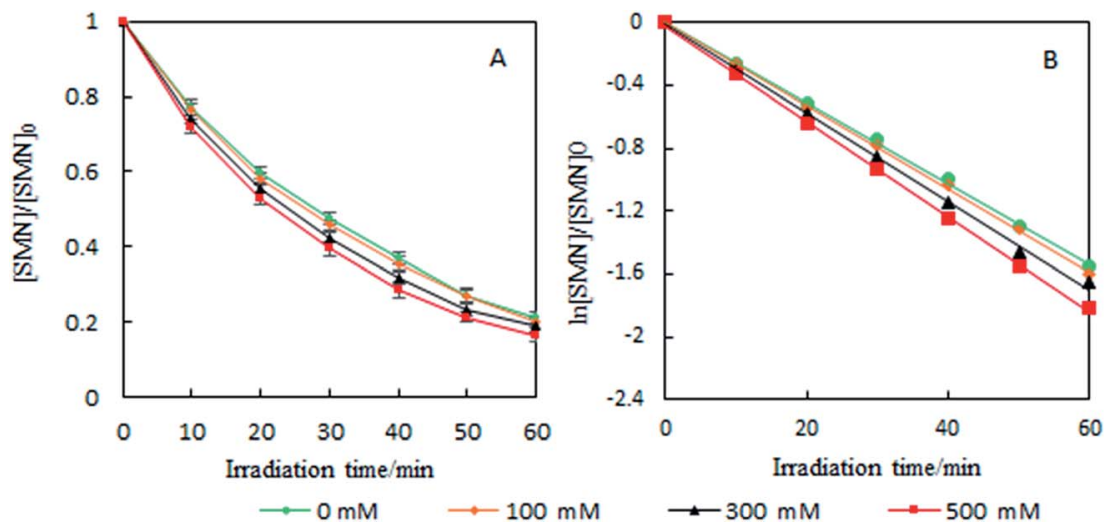

Fig. 5 Effects of $\mathrm{Cl}^{-}$on degradation ratio (A) and kinetics (B) of SMN photolysis.

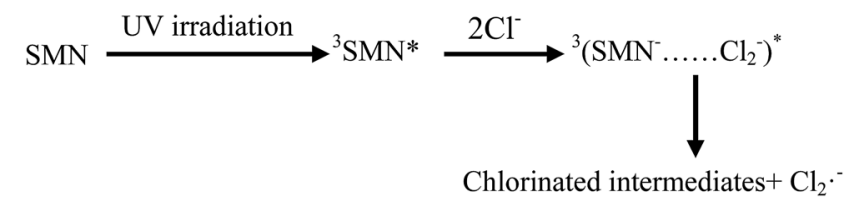

Scheme 2 Reaction pathway of SMN induced by chloride ion. absorption for photons (200-400 nm) by FA could form ${ }^{3} \mathrm{FA}^{*}$, which inhibited the production of ${ }^{3} \mathrm{SMN}^{*}$. Another, the ${ }^{3} \mathrm{FA}^{*}$ was quenched by dissolved oxygen in the water. This process takes ${ }^{3} \mathrm{FA}^{*}$ back to the ground state (FA). FA not only acts as a ROS scavenger, ${ }^{52}$ but also inhibits SMN from absorbing the photons.
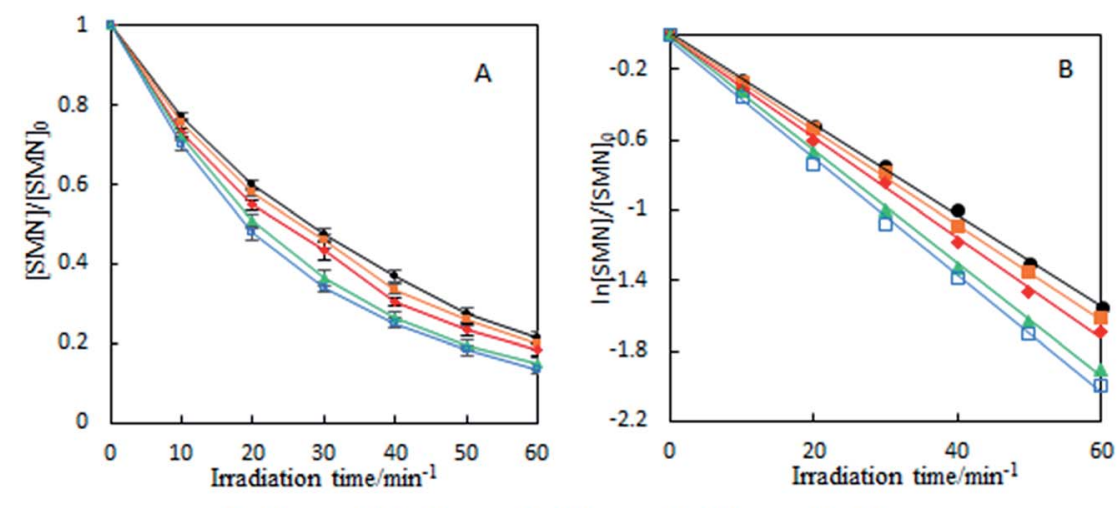

Fig. 6 Effects of $\mathrm{NO}_{3}{ }^{-}$on degradation ratio (A) and kinetics (B) of SMN photolysis.

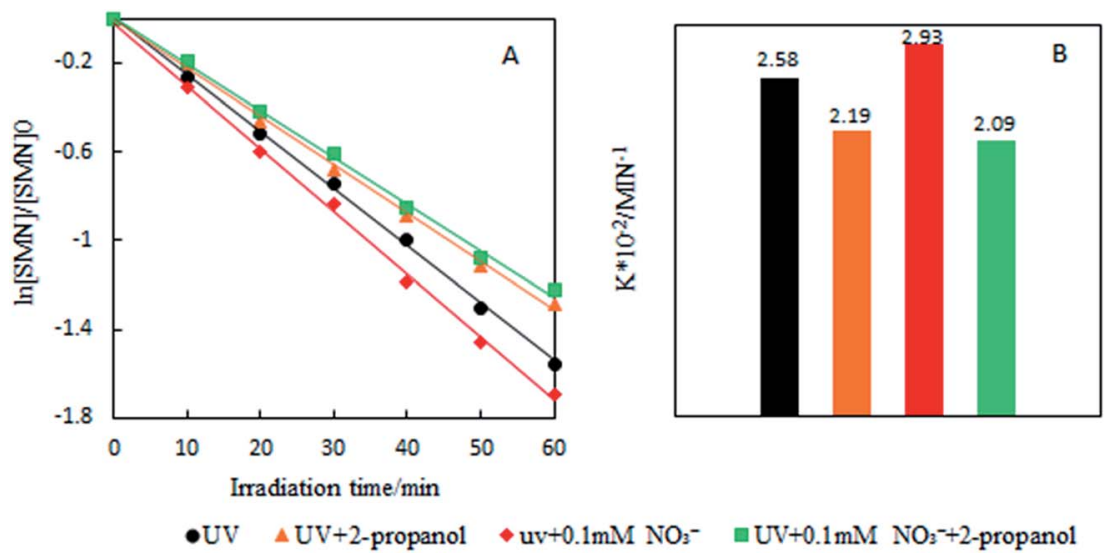

Fig. 7 The $k$ values of SMN photolysis after adding $\mathrm{NO}_{3}{ }^{-}$and 2-propanol. 

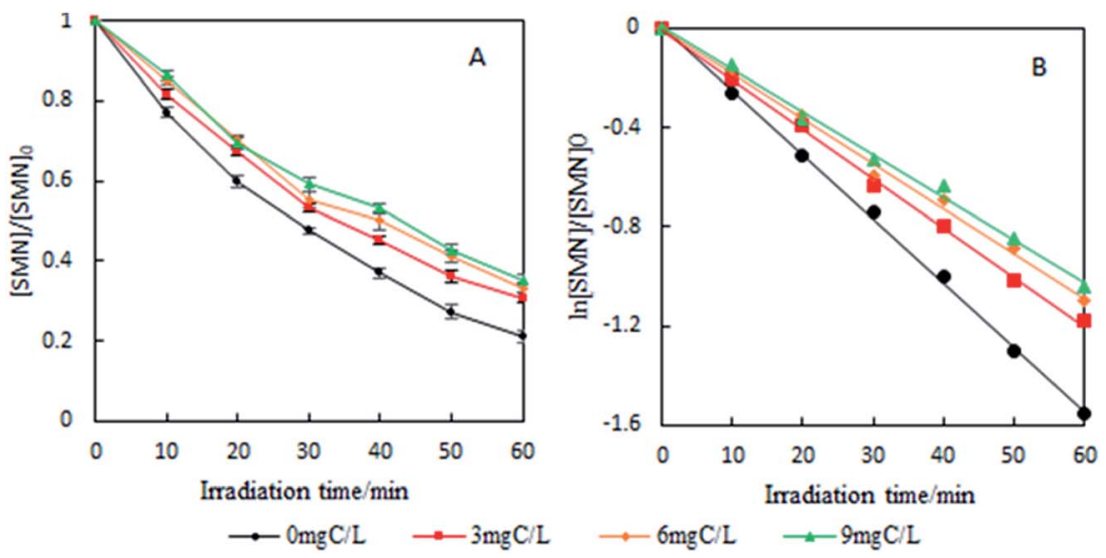

Fig. 8 Effects of FA on degradation ratio (A) and kinetics (B) of SMN photolysis.

\section{Conclusion}

In this study, we investigated the photolytic behaviors of SMN via adding different scavengers to quench active species in pure water. The research indicated that ${ }^{3} \mathrm{SMN}^{*}$ played an important role in SMN photolysis: the main photolytic path of SMN in water was ${ }^{3} \mathrm{SMN}^{*}$ direct photolysis. Moreover, ${ }^{\circ} \mathrm{OH}$ formed by self-sensitized SMN could degrade SMN, which could not be ignored during study of SMN photochemical behaviors. The main photoproducts of SMN were identified by LC-MS/MS, which showed that SMN could not be mineralized although photolysis under UV was effective. Via studying the effects of ordinary soluble substances in natural water on SMN photolysis, we also found that the rate of SMN photolysis was faster in the presence of $\mathrm{Cl}^{-}$and $\mathrm{NO}_{3}{ }^{-}$. The triplet-induced halogenation of SMN increased the ionic strength and reduced the ground state SMN; these were the primary causes of promotion of SMN photolysis by $\mathrm{Cl}^{-}$. More ${ }^{\circ} \mathrm{OH}$ produced in the presence of $\mathrm{NO}_{3}{ }^{-}$ could be of advantage to SMN photolysis. A low concentration of FA in water inhibited SMN photolysis. Competitive absorption of photons by FA with SMN and ROS scavenged by FA were considered to be the main reasons. The environmental risks of intermediates during SMN photolysis in waters must cause concern.

\section{Conflicts of interest}

There are no conflicts of interest to declare.

\section{Acknowledgements}

The authors are grateful for the financial support provided by the Sci-Tech Support Plan Foundation of Sichuan Province, China (Grant No. 2017RZ0035), Scientific Research Foundation of the Education Department of Sichuan Province, China (Grant No. 15ZB0261), Key Project of Technological Innovation of Leshan Normal University, Sichuan Province, China (Grant No. Z1413), Project of Introduction of teachers of Leshan Normal University, Sichuan Province, China (Grant No. Z1517), and
Scientific Research Foundation of Leshan Science \& Technology Bereau, Sichuan Province, China (Grant No. 15ZDYJ0144).

\section{References}

1 W. Baran, E. Adamek, J. Ziemiánska and A. Sobczak, Effects of the presence of sulfonamides in the environment and their influence on human health, J. Hazard. Mater., 2011, 196, 1-15.

2 A. P. S. Batista, F. C. C. Pires and A. C. S. C. Teixeira, The role of reactive oxygen species in sulfamethazine degradation using UV-based technologies and products identification Ana, J. Photochem. Photobiol., A, 2014, 290, 77-85.

3 Q. Q. Zhang, et al., Comprehensive evaluation of antibiotics emission and fate in the river basins of China: source analysis, multimedia modeling, and linkage to bacterial resistance, Environ. Sci. Technol., 2015, 49, 6772-6782.

4 A. K. Sarmah, M. T. Meyer and A. B. Boxall, A global perspective on the use, sales, exposure pathways, occurrence, fate and effects of veterinary antibiotics (VAs) in the environment, Chemosphere, 2006, 65, 725-759.

$5 \mathrm{~K}$. Chen and J. L. Zhou, Occurrence and behavior of antibiotics in water and sediments from the Huangpu River, Shanghai, China, Chemosphere, 2014, 95, 604-612.

6 Y. J. Li, et al., Effects of halide ions on photodegradation of sulfonamide antibiotics: formation of halogenated intermediates, Water Res., 2016, 102, 405-412.

7 A. Shimizu, et al., Ubiquitous occurrence of sulfonamides in tropical Asian waters, Sci. Total Environ., 2013, 452-453.

8 R. Zhang, et al., Occurrence and risk of antibiotics in the coastal aquatic environment of the Yellow Sea. North China, Sci. Total Environ., 2013, 450-451, 197-204.

9 L. J. Zhou, et al., Excretion masses and environmental occurrence of antibiotics in typical swine and dairy cattle farms in China, Sci. Total Environ., 2013, 444, 183-195.

10 A. L. Batt, D. D. Snow and D. S. Aga, Occurrence of sulfonamide antimicrobials in private water wells in Washington County, Idaho, USA, Chemosphere, 2006, 64, 1963-1971. 
11 M. J. García-Galán, et al., Application of fully automated online solid phase extraction-liquid chromatography electrospray-tandem mass spectrometry for the determination of sulfonamides and their acetylated metabolites in groundwater, Anal. Bioanal. Chem., 2011, 399, 795-806.

12 I. Arslan-Alaton, S. Dogruel, E. Baykal and G. Gerone, Combined chemical and biological oxidation of penicillin formulation effluent, J. Environ. Manage., 2004, 73, 155-163.

$13 \mathrm{~K}$. Kümmerer, Antibiotics in the aquatic environment a review part I, Chemosphere, 2009, 75, 417-434.

14 A. G. Trovó, et al., Degradation of sulfamethoxazole in water by solar photo-Fenton. Chemical and toxicological evaluation, Water Res., 2009, 43, 3922-3931.

15 M. J. García-Galán, M. Silvia Díaz-Cruz and D. Barceló, Identification and determination of metabolites and degradation products of sulfonamide antibiotics, Trends Anal. Chem., 2008, 27, 1008-1022.

16 M. J. García-Galán, M. S. Díaz-Cruz and D. Barceló, Combining chemical analysis and ecotoxicity to determine environmental exposure and to assess risk from sulfonamides, Trends Anal. Chem., 2009, 28, 804-819.

17 A. Białk-Bielinska, et al., Ecotoxicity evaluation of selected sulfonamides, Chemosphere, 2011, 85, 928-933.

18 L. H. M. L. M. Santos, et al., Ecotoxicological aspect As related to the presence of pharmaceuticals in the aquatic environment, J. Hazard. Mater., 2010, 175, 45-95.

19 T. W. Tzeng, et al., Photolysis and photocatalytic decomposition of sulfamethazine antibiotics in an aqueous solution with $\mathrm{TiO}_{2}$, RSC Adv. , 2016, 6, 69301-69310.

20 U. Riaz, S. M. Ashraf and J. Kashyap, Enhancement of photocatalytic properties of transitional metal oxides using conducting polymers: A mini review, Mater. Res. Bull., 2015, 71, 75-90.

21 U. Riaz, S. M. Ashraf and A. Ruhela, Catalytic degradation of orange $\mathrm{G}$ under microwave irradiation with a novel nanohybrid catalyst, J. Environ. Chem. Eng., 2015, 3, 20-29.

22 U. Riaz, et al., Sonochemical Facile Synthesis of SelfAssembled Poly(o-phenylenediamine)/Cobalt Ferrite Nanohybrid with Enhanced Photocatalytic Activity, Ind. Eng. Chem. Res., 2016, 55, 6300-6309.

23 U. Riaz and S. M. Ashraf, Synergistic effect of microwave irradiation and conjugated polymeric catalyst in the facile degradation of dyes, RSC Adv., 2014, 4, 47153.

24 X. H. Wang and A. Y. C. Lin, Photo transformation of cephalosporin antibiotics in an aqueous environment results in higher toxicity, Environ. Sci. Technol., 2012, 46, 12417-12426.

25 S. Yan and W. Song, Photo-transformation of pharmaceutically active compounds in the aqueous environment: a review, Environ. Sci.: Processes Impacts, 2014, 16, 697-720.

26 L. E. Jacobs, et al., Fulvic acid mediated photolysis of ibuprofen in water, Water Res., 2011, 45, 4449-4458.

27 M. M. Kelly and W. A. Arnold, Direct and indirect photolysis of the phytoestrogens genistein and daidzein, Environ. Sci. Technol., 2012, 46, 5396-5403.
$28 \mathrm{~J} . \mathrm{Li}$, et al., Effects of nitrate and humic acid enrofloxacin photolysis in an aqueous system under three light condition: kinetics and mechanism, Environ. Chem., 2014, 11, 333-340.

29 R. R. Chowdhury, P. A. Charpentier and M. B. Ray, Photodegradation of 17-estradiol in aquatic solution under solar irradiation: kinetics and influencing water parameters, J. Photochem. Photobiol., A, 2011, 219, 67-75.

30 T. Zeng and W. A. Arnold, Pesticide photolysis in prairie potholes: probing photo-sensitized processes, Environ. Sci. Technol., 2013, 47, 6735-6745.

31 A. G. Trovó, et al., Photodegradation of sulfamethoxazole in various aqueous media: Persistence, toxicity and photoproducts assessment, Chemosphere, 2009, 77, 12921298.

32 L. K. Ge, et al., New insights into the aquatic photochemistry of fluoroquinolone antibiotics: Direct photodegradation, hydroxyl-radical oxidation, and antibacterial activity changes, Sci. Total Environ., 2015, 527-528, 12-17.

33 M. Sturini, et al., Sunlight-induced degradation of fluoroquinolones in wastewater effluent: Photoproducts identification and toxicity, Chemosphere, 2015, 134, 313318.

34 S. J. Jiao, et al., Aqueous photolysis of tetracycline and toxicity of photolytic products to luminescent bacteria, Chemosphere, 2008, 73, 377-382.

35 Y. Chen, et al., Photolysis of chlortetracycline in aqueous solution: Kinetics, toxicity and products, J. Environ. Sci., 2012, 24, 254-260.

36 V. K. Balakrishnan, K. A. Terry and J. Toito, Determination of sulfonamide antibiotics in wastewater: a comparison of solid phase micro extraction and solid phase extraction methods, J. Chromatogr. A, 2006, 1131, 1-10.

37 L. Sun, et al., Analysis of sulfonamides in environmental water samples based on magneticmixed hemimicelles solid-phase extraction coupled with HPLC-UV detection, Chemosphere, 2009, 77, 1306-1312.

38 R. Nassar, et al., Photodegradation of sulfamethazine, sulfamethoxypiridazine, amitriptyline, and clomipramine drugs in aqueous media, J. Photochem. Photobiol., A, 2017, 336, 176-182.

39 M. K. Li, et al., Sulfamethazine degradation in water by the VUV/UV process: Kinetics, mechanism and antibacterial activity determination based on a mini fluidic VUV/UV photoreaction system, Water Res., 2017, 108, 348-355.

40 D. E. Moore, Mechanisms of photosensitization by phototoxic drugs, Mutat. Res., 1998, 422, 165-173.

41 S. Canonica, U. Jans, K. Stemmler and J. Hoigne, Transformation kinetics of phenols in water: Photosensitization by dissolved natural organic material and aromatic ketones, Environ. Sci. Technol., 1995, 29, 1822-1831.

42 J. Wenk, U. Gunten and S. Canonica, Effect of dissolved organic matter on the transformation of contaminants induced by excited triplet states and the hydroxyl radical, Environ. Sci. Technol., 2011, 45, 1334-1340. 
43 M. M. Dong and F. L. Rosario-Ortiz, Photochemical formation of hydroxyl radical from effluent organic matter, Environ. Sci. Technol., 2012, 46, 3788-3794.

44 Y. J. Li, et al., Photodegradation mechanism of sulfonamides with excited triplet state dissolved organic matter: A case of sulfadiazine with 4-carboxybenzophenone as a proxy, $J$. Hazard. Mater., 2015, 290, 9-15.

45 S. Bahnmüller, U. Gunten and S. Canonica, Sunlightinduced transformation of sulfadiazine and sulfamethoxazole in surface waters and wastewater effuents, Water Res., 2014, 57, 183-192.

46 G. J. Janz, B. G. Oliver, G. R. Lakshminarayanan and G. E. Mayer, Electrical conductance, diffusion, viscosity, and density of sodium nitrate, sodium perchlorate, and sodium thiocyanate in concentrated aqueous solutions, $J$. Phys. Chem., 1970, 74, 1285-1289.

47 E. R. Nightingale Jr, Viscosity of aqueous sodium perchlorate solutions, J. Phys. Chem., 1959, 63, 742-743.
48 S. Canonica, B. Hellrung and J. Wirz, Oxidation of phenols by triplet aromatic ketones in aqueous solution, J. Phys. Chem. A, 2000, 104, 1226-1232.

49 D. Jornet, et al., Experimental and theoretical studies on the mechanism of photochemical hydrogen transfer from 2aminobenzimidazole to $\mathrm{np}$ and $\mathrm{pp}$ aromatic ketones, $J$. Phys. Chem. B, 2010, 114, 11920-11926.

50 H. S. Yang, X. Yang, L. Xu and A. Q. Zhang, The progress of humic substances photochemistry in natural waters, Photogr. Sci. Photochem., 2004, 2, 22.

$51 \mathrm{~J}$. Werner, et al., Environmental photochemistry of tylosin: efficient, reversible photoisomerization to a less active isomer, followed by photolysis, J. Agric. Food Chem., 2007, 55, 7062-7068.

52 Y. Li, J. F. Niu and W. L. Wang, Photolysis of Enrofloxacin in aqueous systems under simulated sunlight irradiation: Kinetics, mechanism and toxicity of photolysis products, Chemosphere, 2011, 85, 892-897. 